\title{
Fingertip skin conformance accounts, in part, for differences in tactile spatial acuity in young subjects, but not for the decline in spatial acuity with aging
}

\author{
FRANCISCO VEGA-BERMUDEZ and KENNETH O. JOHNSON \\ Johns Hopkins University, Baltimore, Maryland
}

\begin{abstract}
The ability of the skin to conform to the spatial details of a surface or an object is an essential part of our ability to discriminate fine spatial features haptically. In this study, we examined the extent to which differences in tactual acuity between subjects of the same age and between younger and older subjects can be accounted for by differences in the properties of the skin. We did so by measuring skin conformance and tactile spatial acuity in the glabrous skin at the fingertip in 18 younger (19-36 years old) and 9 older (61-69 years old) subjects. Skin conformance was measured as the degree to which the skin invaded the spaces in the psychophysical stimuli. There were severalfindings. First, skin conformance accounted for $50 \%$ of the variance in our measure of tactile spatial acuity (the threshold for grating orientation discrimination) between the younger subjects. The subjects with more compliant skin had substantially lower thresholds than did the subjects with stiffer skin. Second, the skin of the younger subjects was more compliant across than along the skin ridges, and this translated into significantly greater performance when the gratings were oriented along than when oriented across the skin. Third, skin conformance was virtually identical in the younger and the older subjects. Consequently, skin conformance cannot account for the loss of spatial acuity reported in earlier studies and confirmed in this study. We infer that the loss must be neural in origin.
\end{abstract}

It is well known that tactile spatial acuity at the fingertips (the ability to resolve fine spatial detail) varies significantly between subjects of the same age (Vega-Bermudez \& Johnson, 2001; Vega-Bermudez, Johnson, \& Hsiao, 1991) and between younger and older subjects (Stevens \& Choo, 1996; Stevens \& Patterson, 1995; Vega-Bermudez \& Johnson, 2002), but the reason for this variation is not known. The variation is not due to the testing methods, because the grating orientation discrimination measure used in several of these studies has been shown to be highly repeatable (Van Boven \& Johnson, 1994a). Other possibilities are differences in the central pathways leading to tactile perception, differences in afferent innervation density, which has a significant effect on spatial acuity (Van Boven \& Johnson, 1994b), or differences in skin properties.

The skin property that is critical for the recognition of small objects or surface features is the skin's ability to conform to the object or the surface. If the skin fails to bend and follow the surface features or does so poorly, the mechano-

This work was supported by a fellowship from the Robert Wood Johnson Foundation (Grants 037213 and 038559) and by NIH Grants NS 18787 and 38034 . Correspondence concerning this article should be addressed to F. Vega-Bermudez, 338 Krieger Hall, Zanvyl Krieger Mind/Brain Institute, Johns Hopkins University, 3400 N. Charles Street, Baltimore, MD 21218 (e-mail: fvega@jhu.edu).

Note-This article was accepted by the previous editorial team, headed by Neil Macmillian. receptors responsible for conveying the spatial form of the stimulus cannot do so. The skin's ability to conform to a spatial pattern is functionally analogous to the eye's ability to focus an image on the retina. A major source of variation in the uncorrected visual acuity of young adults is variation in their ability to focus an image (i.e., variation in their lenses). The question addressed here is whether variation in skin conformance contributes to variation in tactile acuity.

The mechanics of contact between the skin and an object are complex (K. L. Johnson, 1985). In the only previous study (Woodward, 1993) in which measures of tactile spatial discrimination were compared with skin properties, the indentation produced by $10 \mathrm{~g}$ of force (i.e., compliance) was used as a measure, and no relationship was found. However, compliance does not measure the degree to which the skin bends and molds to the stimulus, which is the critical issue. A rigid surface, such as a plastic computerkey, can be highly compliant. There is no conventional measure of conformance of which we are aware. Because we used mechanical gratings to measure tactile spatial acuity, we adopted the degree to which the skin invaded a slot exactly like the slots in the test grating as a measure of conformance.

In the present investigation, we measured skin conformance and the grating orientation threshold (GOT) the finest grating that allowed reliable grating orientation discrimination - in the fingertips of subjects 19-69 years old. The study had two main aims. The first was to see whether the relatively large variation in tactile spatial acu- 
ity between subjects of the same age is due to differences in skin conformance. The second was to see whether the progressive decline in tactile acuity with age is related to a progressive change in the skin's ability to conform to the shape of a stimulus.

We confined our study to the tip of the index finger because it is the area of greatest tactile acuity and, like the fovea, most critical for fine form perception (VegaBermudez \& Johnson, 2001). Tactile spatial acuity at the fingertip is very close to the limits imposed by neural innervation density (Phillips, Johnson, \& Browne, 1983), and it may also be limited by skin mechanics - the skin's ability to conform.

We used gratings in this study because GOTs are closely correlated with the ability to discriminate complex shapes, such as raised letters (Vega-Bermudez \& Johnson, 2001), they have been used in many studies (Craig \& Lyle, 2001; Sathian \& Zangaladze, 1996; Schlereth, Magerl, \& Rolf-Detlef, 2001), and the test-retest variation for individual subjects over many testing sessions is only about $10 \%$ of the mean threshold value (Van Boven \& Johnson, 1994a). We measured the skin's invasion of the grating slots because that is a direct measure of conformance.

\section{METHOD}

The subjects consisted of a younger group of 18 ( 5 females and 13 males) right-handed subjects, 19-36 years old, and an older group of 9 right-handed subjects (5 females and 4 males), 61-69 years old. None of the 27 subjects had a history of nerve injury, finger trauma, or learning disability, and the finger pad tested was free of calluses. Consent was obtained from each subject, and the Human Subjects Institutional Review Board of Johns Hopkins University approved the experiments.

\section{Grating Orientation Discrimination}

The subjects' ability to discriminate the orientation of a mechanical square-wave grating was tested with JVP dome gratings (Stoelting Co., Wood Dale, IL) with equal groove and ridge widths $(0.35$, $0.5,0.75,1.0,1.2,1.5,2.0$, and $3.0 \mathrm{~mm}$ ) plus two domed gratings, made for this study, with groove and ridge widths of 4.0 and $5.0 \mathrm{~mm}$. A grating was applied for $1-5 \mathrm{sec}$ to the distal one third of the finger pad, with the grooves either along or across the finger axis, and the subjects were required to state the orientation. At this location, the skin ridges are orthogonal to the finger axis, so the two grating orientations were also parallel and orthogonal to the skin ridges.

The experiment started with a grating that was easily discriminated $(3.0-\mathrm{mm}$ grooves for younger subjects, $5.0-\mathrm{mm}$ grooves for older subjects). All 9 older subjects judged the grating with $5.0-\mathrm{mm}$ grooves to be easily resolved. Each grating was presented 20 times (10 times with ridges oriented along and 10 times across the finger axis) before moving to the next, finer grating. A computer-generated random table determined the orientation for each trial. Gradually, finer gratings were presented until the subjects' performance approached chance (50\% correct responses). The GOT was defined as the groove width at which the performance was $75 \%$ correct. Interpolation between gratings spanning the $75 \%$ correct responses was used unless performance was exactly $75 \%$ for a particular grating.

\section{Skin Conformance Measurement}

Because the psychophysical stimuli were mechanical gratings with bars and slots, skin conformance was measured as the depth to which the skin invaded a slot just like the grating slots. Because most GOTs fall in the range from 1 to $3 \mathrm{~mm}$, slots 1,2 , and $3 \mathrm{~mm}$ wide were used. The invasion was measured with a 0.5 -mm-diameter pin that protruded through the center of the slot to rest just above the plate. The pin was mounted on a balance beam with counterweights adjusted to produce a reaction force of $5 \mathrm{mg}$. The pin was depressed as the skin entered the slot, which raised a mirror on the other end of the balance beam. The mirror displacement (and, thus, the skin invasion) was measured with a laser Doppler displacement meter (2-nm resolution; Optodyne Inc., Compton, CA). The entire apparatus was mounted on a balance scale (Dial-o-gram balance; Ohaus Co., Florham Park, NJ) that was set to maintain a reaction force of $80 \mathrm{~g}$ when depressed. Preliminary tests showed that people use 30-130 g when pressing with a force of their own choosing and that force variations in this range have a minor effect on conformance (see the Results section).

Before each skin conformance measurement, the zero-reference displacement was obtained by placing a perfectly flat block over the slot (a gauge block; Phase II Co., Carlstadt, NJ) so that the top of the pin was flush with the surface of the contact plate ( \pm 0.030 microns). Skin invasion into the slot was sampled every $2 \mathrm{msec}$, beginning $3-5 \mathrm{sec}$ after initial skin contact (the time required for finger placement and for the force to stabilize at $80 \mathrm{~g}$ ) and continuing for $5 \mathrm{sec}$. These samples were used to calculate the average skin invasion. Repeated measures of skin conformance showed that trial-to-trial variation from all sources was \pm 2 microns $(S D)$.

The skin region tested (distal one third of the finger pad), the slot widths $(1,2$, and $3 \mathrm{~mm}$ ), their orientations (parallel and perpendicular to the skin ridges), the timing ( $5 \mathrm{sec}$ of contact, $10-20 \mathrm{sec}$ between measurements), and the contact force were all selected to match as closely as possible the stimulus conditions in the grating orientation discrimination experiments.

Each measure of conformance was obtained by placing the subject's finger over the slot at approximately $45^{\circ}$ relative to the plate, so that only the distal one third of the finger pad contacted the plate. We used the distal one third of the finger pad because this was the region tested psychophysically and because the skin ridges run in a single direction perpendicular to the long axis of the finger. Care was taken to use the same skin region for the measurement of conformance and the psychophysical testing. Each conformance measurement was repeated 5 times. Thus, each subject was tested 30 times ( 3 slot widths $\times 2$ orientations $\times 5$ repetitions).

\section{RESULTS}

\section{Force Effects}

The effects of contact force on skin invasion were measured in 4 subjects (Figure 1). Forces of 40, 80, and $120 \mathrm{~g}$ were selected to span the range of forces used by the subjects asked to press the gratings with whatever force felt most comfortable (see the Method section). The results show that the skin is highly conformable. Forces less than $40 \mathrm{~g}$ produce a large fraction of the conformation (slot invasion) of which the skin is capable. The effect of additional force depends on the width of the slot. A threefold increase in force from 40 to $120 \mathrm{~g}$ produced $107 \%, 57 \%$, and $30 \%$ increases in skin invasion into the 1-, 2-, and 3-mm slots, respectively, when the slots were parallel to the skin ridges. The comparable percentages when the slots were perpendicular to the skin ridges were nearly identical $(100 \%, 54 \%$, and $33 \%)$. Because force had a secondary effect on conformance, all the subjects were tested at a single force, $80 \mathrm{~g}$, near the middle of the range of typical forces.

\section{GOT and Conformance in Younger Subjects}

The data collected from the younger group are shown in Table 1. The average GOT for these subjects was $1.21 \mathrm{~mm}$ 


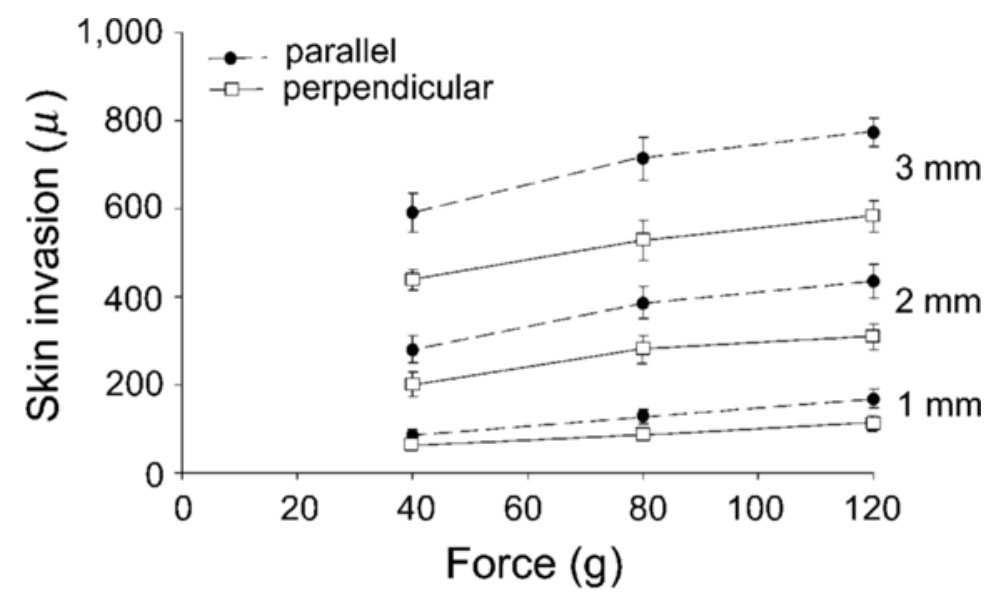

Figure 1. Skin invasion versus force in 4 young subjects. Skin invasion was measured as the maximum skin protrusion into a slot whose width is illustrated at the right of each graph. Dashed lines represent skin invasion when the slot was oriented parallel to the skin ridges; solid lines, when it was perpendicular to the skin ridges. Error bars represent the standard error of the mean for each point.

(0.08 mm SE). The depth to which the skin invaded each slot for slots oriented parallel and perpendicular to the skin ridges is shown for each subject. A three-way analysis of variance (ANOVA) with subjects, slot width, and slot orientation as factors showed that all three had a highly significant effect on skin invasion [subjects, $F(17,87)=7.69$; slot width, $F(2,87)=1,006$; slot orientation, $F(1,87)=$ $30.16 ; p<.001$ for all three]. The skin conformance data for the younger subjects are plotted in Figure 2, which shows that there is a progressively increasing skin invasion with increasing slot size and that, on average, the skin ridges have a small but significant effect on conformance. The average differences in skin invasion between the parallel and the perpendicular orientations were 14, 62, and 106 microns for the 1-, 2-, and 3-mm slots, respectively.

The relationship between skin conformance and spatial acuity is illustrated in Figure 3. The index of skin conformance for each subject is the slope of the linear regression of skin invasion on slot size. The mean slope is $0.293 \mathrm{~mm}$ of skin invasion per millimeter increase in slot size $(0.271$ perpendicular and 0.317 parallel to skin ridges). The subjects with greater skin conformance had lower thresholds, and the correlation, -.704 , accounted for $50 \%$ of the variance in the thresholds between subjects (Hoetelling's transform to normality, $z=3.42, p<.001$; Sokal \& Rohlf, 1969, pp. 516-518).

\section{Effect of Orientation}

The data so far suggest a relationship between conformance and the ability to discriminate grating orientation. If that is true, the difference in conformance with grating orientation should translate into a difference in subjects' performance. Because the skin conforms to the stimulus more when the skin ridges and gratings are parallel to one another, subjects should recognize that grating orientation more accurately. Figure 4 shows that, in fact, the subjects were more accurate when the gratings were parallel to the skin ridges than when they were perpendicular. Over all presentations, the subjects judged the orientations of parallel and perpendicularorientations with $88.7 \%$ and $79.7 \%$ accuracy, and the difference was significant $[t(17)=2.71$, $p=.015]$. If only the presentations of gratings close to threshold are used (the gratings immediately above and below threshold), the subjects' scores were $81.5 \%$ and $67.5 \%$ for parallel and perpendicular orientations $[t(17)=$ $3.22, p=.005]$.

\section{Response Bias}

The greater accuracy when the gratings were parallel to the skin ridges (oriented across the finger axis) could have been due to a response bias. To control for this possibility, all the subjects judged the orientation of a very fine grating $(0.35-\mathrm{mm}$ slots and bars) for which performance was close to chance. If there was a general bias toward calling orientations orthogonal to the finger axis (parallel to the skin ridges), it should apply to these stimuli as well, but it did not: Gratings parallel and perpendicular to the skin ridges were discriminated correctly on $53.2 \%$ and $56.8 \%$ of the trials, and the difference was not significant $[t(17)=$ $1.36, p=.19]$. Thus, we infer that the difference in accuracy was due to a difference in skin conformance and not to bias.

\section{GOT and Skin Conformance in Older Subjects}

Next, we inquired whether differences in skin conformance could account for the decline in performance in older subjects, which has been widely reported (Stevens \& Choo, 1996; Vega-Bermudez \& Johnson, 2002) and which we confirmed in this study. The results for the 9 older subjects, 60-69 years in age, are shown in Table 2. As was expected, the older subjects had poorer spatial acuity than 
Table 1

Skin Conformance and Tactile Acuity in Younger Subjects

\begin{tabular}{|c|c|c|c|c|c|c|c|c|c|}
\hline \multirow[b]{2}{*}{$\underline{\text { Subject }}$} & \multirow[b]{2}{*}{ Age } & \multirow[b]{2}{*}{ Sex } & \multirow[b]{2}{*}{ GOT } & \multicolumn{3}{|c|}{ Parallel } & \multicolumn{3}{|c|}{ Perpendicular } \\
\hline & & & & $1 \mathrm{~mm}$ & $2 \mathrm{~mm}$ & $3 \mathrm{~mm}$ & $1 \mathrm{~mm}$ & $2 \mathrm{~mm}$ & $3 \mathrm{~mm}$ \\
\hline A1 & 19 & $\mathrm{~m}$ & 1.10 & 125 & 385 & 755 & 97 & 303 & 596 \\
\hline $\mathrm{A} 2$ & 21 & $\mathrm{f}$ & 0.86 & 193 & 498 & 946 & 198 & 448 & 945 \\
\hline A3 & 21 & $\mathrm{~m}$ & 0.96 & 140 & 423 & 740 & 125 & 399 & 702 \\
\hline A4 & 21 & $\mathrm{~m}$ & 1.26 & 146 & 418 & 792 & 124 & 268 & 555 \\
\hline A5 & 22 & $\mathrm{~m}$ & 0.88 & 138 & 431 & 914 & 150 & 412 & 751 \\
\hline A6 & 22 & $\mathrm{~m}$ & 0.95 & 181 & 502 & 1,016 & 190 & 492 & 803 \\
\hline A7 & 22 & $\mathrm{~m}$ & 1.00 & 135 & 440 & 751 & 96 & 253 & 544 \\
\hline A8 & 23 & $\mathrm{f}$ & 1.00 & 192 & 543 & 922 & 219 & 497 & 854 \\
\hline A9 & 23 & $\mathrm{~m}$ & 1.20 & 96 & 342 & 709 & 73 & 251 & 596 \\
\hline A10 & 24 & $\mathrm{~m}$ & 1.75 & 140 & 466 & 811 & 112 & 356 & 652 \\
\hline A11 & 25 & $\mathrm{~m}$ & 1.10 & 255 & 553 & 873 & 195 & 371 & 684 \\
\hline A 12 & 25 & $\mathrm{f}$ & 1.00 & 148 & 429 & 792 & 145 & 420 & 745 \\
\hline A13 & 26 & $\mathrm{f}$ & 1.09 & 137 & 391 & 739 & 126 & 417 & 753 \\
\hline A14 & 27 & $\mathrm{~m}$ & 1.50 & 103 & 374 & 707 & 103 & 304 & 579 \\
\hline A 15 & 31 & $\mathrm{~m}$ & 1.80 & 210 & 407 & 696 & 276 & 372 & 664 \\
\hline A16 & 31 & $\mathrm{~m}$ & 1.75 & 170 & 383 & 635 & 153 & 349 & 613 \\
\hline A17 & 35 & $\mathrm{~m}$ & 1.70 & 160 & 397 & 701 & 118 & 376 & 588 \\
\hline A 18 & 36 & f & 0.89 & 94 & 324 & 683 & 94 & 309 & 659 \\
\hline Mean & 25.2 & & 1.21 & 154 & 428 & 788 & 144 & 367 & 682 \\
\hline
\end{tabular}

Note-GOT, grating orientation threshold.

did the younger subjects (Figure 5). The mean threshold in the older group, $1.97 \mathrm{~mm}$, was significantly greater than the mean for the younger group, $1.2 \mathrm{~mm}[t(25)=4.06$, $p<.001]$. Although the GOT varied widely in this group, there was no significant relationship between GOT and skin conformance (correlation, .04). Thus, skin conformance accounted only for $<1 \%$ of the variance. This correlation was significantly different from the value for the younger subjects (Hoetelling's transform to normality, $z=$ $2.03, p=.02$; Sokal \& Rohlf, 1969, pp. 516-518). Unlike the younger subjects, there was no relationship between slot orientation and conformance $[t(8)=0.43, p=.68]$, and there was no significant difference in skin conformance between the younger and the older subjects [three-way ANOVA with age group, slot width, and orientation as factors; $F$ ratio for age group effect, $F(1,157)=0.238, p=$ .63 ]. These similarities are illustrated in Figure 6.

\section{DISCUSSION}

The aim of this study of the correlation between grating orientation discrimination and skin conformance was to further our understanding of the mechanisms of tactile form perception. There were two main findings. First, skin conformance in the fingertip had a substantial effect on acuity within the younger group. The subjects with more conformant skin generally had lower orientation thresholds. The same was true of the subjects in the older group, but the effect accounted for a negligible fraction of the variance. Second, skin conformance in the older subjects was very similar to that in the younger subjects, even though their spatial acuity was much poorer. These data suggest that skin conformance and aging have significant but independent effects on spatial acuity. An interpretation that accounts for these data is that the decline in acuity with age is due to neural effects that vary between subjects, which explains why skin conformance accounted for less of the variance between the older subjects.

There were two secondary findings. First, the skin is highly conformant. Most of the conformance of which the skin is capable occurs at forces less than $40 \mathrm{~g}$. This accounts for the relatively small effects of contact force on tactile

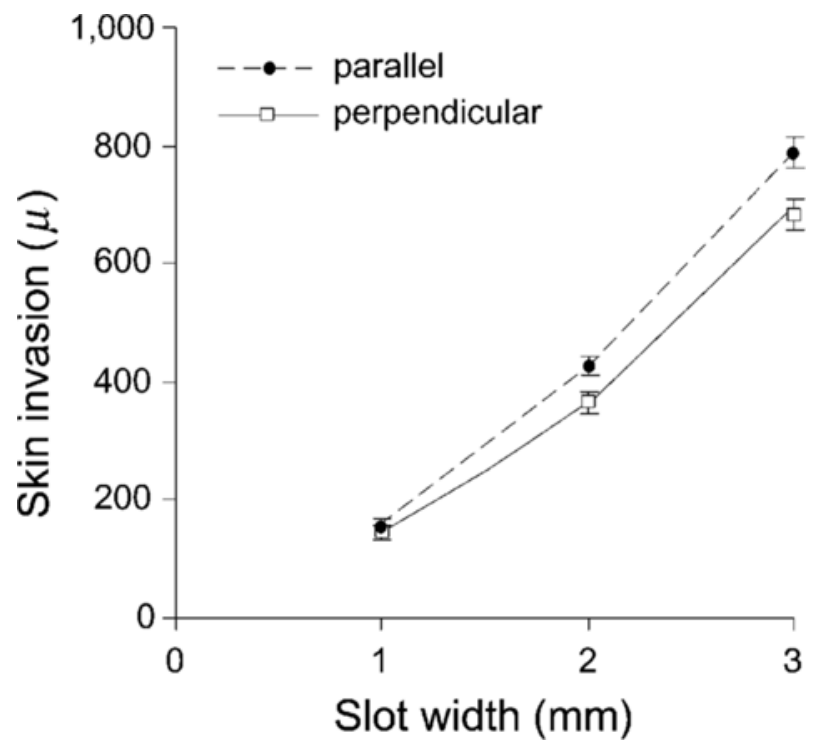

Figure 2. Skin invasion versus slot width for all 18 younger subjects at $80 \mathrm{~g}$ of force. Dashed lines represent skin invasion when the slot was oriented parallel to the skin ridges; solid lines, when it was perpendicular to the skin ridges. Error bars represent the standard error of the mean for each point. 


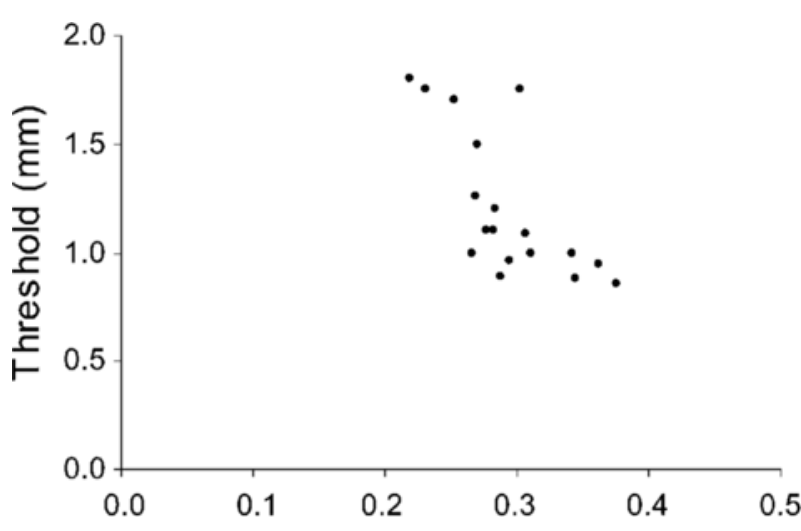

Figure 3. Grating orientation discrimination threshold versus skin conformance for the $\mathbf{1 8}$ young subjects. The threshold is the grating gap and groove size that produced $75 \%$ correct responses. Conformance is defined as the slope of the regression of skin invasion on slot width for each subject. The correlation between the two is $-\mathbf{. 7 0 4}$.

spatial perception and on the neural responses to spatial stimuli, as will be discussed below. Second, when the gratings were oriented so the skin was more conformant (parallel to the skin ridges), the younger subjects judged orientation more accurately. This corroborates the observation of a general correlation between skin conformance and acuity.

\section{Acuity and Skin Conformance}

Woodward (1993) used compliance, defined as the skin indentation produced by $10 \mathrm{~g}$ of force, and found no relationship between skin compliance and tactile acuity measured with two-point discrimination and a gap detection task. There are several explanations for the differences between her findings and ours. First, she measured compliance, not conformance. The ability of the skin to conform to the shape of the stimulus is clearly important for transducing the spatial form of the stimulus. There is no comparable, direct logic linking compliance with spatial acuity. Skin that has lost its collagen and elastin and, therefore, its ability to conform to a stimulus might still be quite compliant (e.g., because it overlies a bed of fatty tissue). Second, she lumped 104 subjects from 18 to 84 years of age into a single group. Even if compliance were correlated with acuity in young subjects, a correlation with acuity in the larger group would be lost. We showed, in the present study, that changes in skin mechanics cannot account for the large and variable loss in tactile spatial acuity. If we had lumped our younger and older subjects, we would have found no significant relationship between conformance and acuity. Third, Woodward measured skin compliance as the mean indentation produced by 8 presentations of two-point stimuli (separation range, $0.0-2.5 \mathrm{~mm}$ in $0.5-\mathrm{mm}$ increments) and 13 presentations of gaps ranging from 0.0 to $4.5 \mathrm{~mm}$. Averaging over 21 different stimuli and size combinations may have obscured any relationship between compliance and acuity. Fourth, at least one of her measures of acuity (the two-point limen) is poorly suited to measuring acuity, and it produces highly variable measures between and within subjects (Craig \& Johnson, 2000).

\section{Neural Responses and Mechanical Anisotropy}

Our observation that skin conformance is anisotropic is in agreement with work by Phillips and Johnson (1981a), who found that cutaneous mechanoreceptors represent the spatial details of a grating more effectively when the bars and slots are parallel to the skin ridges than when they are perpendicular. They suggested that "the dermal ridges may act like parallel rods in a sheet, making it stiff in one direction and flexible in the other" (Phillips \& Johnson, 1981b, p. 1218). Wheat and Goodwin (2000) obtained essentially the same result in a more extensive study. They found that mechanoreceptors respond to a slot in a surface about twice as vigorously when a slot is parallel to the skin ridges than when it is orthogonal. The obvious interpretation of these results is that the mechanoreceptors represent the slots in a grating more effectively when the bars and slots are parallel to the skin ridges, because the skin is more conformant in that orientation. We believe that this accounts for the subject's greater accuracy in identifying gratings when they were parallel to the skin ridges.

\section{Acuity and Mechanical Anisotropy}

The relationship between stimulus orientation and acuity has been studied by several investigators (Craig, 1999; Essock, Krebs, \& Prather, 1992, 1997; Stevens \& Patterson, 1995; Wheat \& Goodwin, 2000). The results vary substantially between studies. The differences are accounted for

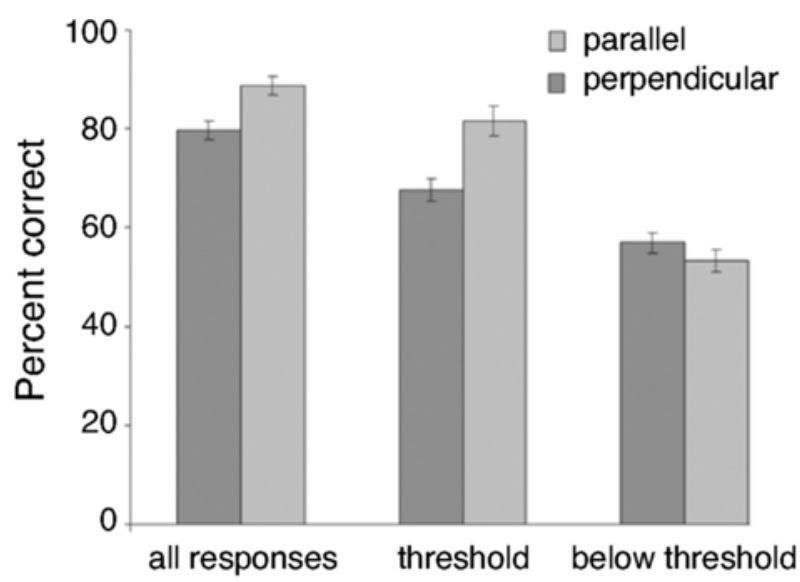

Figure 4. Percentages of correct responses for gratings oriented parallel (light gray) and perpendicular (dark gray) to the skin ridges. Values are shown for all responses, for responses near threshold (responses to the one or two grating gap and groove widths closest to threshold), and for responses below threshold (all gratings with groove and bar widths less than threshold). Bars represent standard errors of the mean. 
Table 2

Skin Conformance and Tactile Acuity in Older Subjects

\begin{tabular}{|c|c|c|c|c|c|c|c|c|c|}
\hline \multirow[b]{2}{*}{ Subject } & \multirow[b]{2}{*}{ Age } & \multirow[b]{2}{*}{ Sex } & \multirow[b]{2}{*}{ GOT } & \multicolumn{3}{|c|}{ Parallel } & \multicolumn{3}{|c|}{ Perpendicular } \\
\hline & & & & $1 \mathrm{~mm}$ & $2 \mathrm{~mm}$ & $3 \mathrm{~mm}$ & $1 \mathrm{~mm}$ & $2 \mathrm{~mm}$ & $3 \mathrm{~mm}$ \\
\hline B1 & 61 & $\mathrm{~m}$ & 1.37 & 136 & 473 & 820 & 100 & 361 & 686 \\
\hline B2 & 67 & $\mathrm{~m}$ & 2.50 & 119 & 370 & 787 & 132 & 423 & 764 \\
\hline B3 & 68 & $\mathrm{f}$ & 2.50 & 142 & 345 & 755 & 92 & 326 & 847 \\
\hline B4 & 69 & $\mathrm{~m}$ & 2.00 & 80 & 280 & 556 & 109 & 254 & 486 \\
\hline B5 & 69 & $\mathrm{f}$ & 1.75 & 99 & 383 & 893 & 206 & 489 & 1,076 \\
\hline B6 & 63 & $\mathrm{f}$ & 2.50 & 141 & 418 & 822 & 104 & 570 & 805 \\
\hline B7 & 65 & $\mathrm{f}$ & 1.67 & 166 & 398 & 719 & 118 & 349 & 660 \\
\hline B8 & 61 & $\mathrm{f}$ & 1.58 & 184 & 471 & 882 & 160 & 468 & 848 \\
\hline B9 & 66 & $\mathrm{~m}$ & 1.88 & 124 & 392 & 685 & 132 & 420 & 813 \\
\hline Mean & 65.4 & & 1.97 & 132 & 392 & 769 & 128 & 407 & 776 \\
\hline
\end{tabular}

Note-GOT, grating orientation threshold.

by differences in the stimuli and how and where they were applied.

Wheat and Goodwin (2000) applied gratings to the distal part of the fingerpad, where the skin ridges are orthogonal to the finger axis, and they asked subjects to discriminate gratings (ridge width, $1.5 \mathrm{~mm}$; slot width, $0.75 \mathrm{~mm}$ ) from smooth surfaces. The subjects in their study, like those in ours, detected the gratings more readily when the ridges and slots were orthogonal to the finger axis (parallel to the skin ridges) than when they were parallel (orthogonal to the skin ridges).

In contrast, Craig (1999) used nearly identical stimuli and found no relationship with orientation relative to the finger axis, but he applied the stimuli at a more proximal location, where the skin ridges are circular. At this location, the skin conformance to the gratings would probably have been the same whether the gaps were oriented parallel or perpendicular to the finger axis.

Other studies have shown differences in tactile discrimination depending on stimulus orientation, but because they did not specify the stimulus location, it is impossible to say whether they are consistent with the anisotropy that we have reported here. Stevens and Patterson (1995) found stimulus orientation differences in two tests of discrimination on the fingertip. In one, subjects judging the orientation of two points were substantially more accurate when the points were oriented along the finger axis. In the other, subjects discriminated an edge from two points (separation equaling the line length) and were slightly better when the two were oriented across the finger axis. Essock et al. $(1992,1997)$ used stimuli similar to those in Wheat and Goodwin (2000) and Craig (1999) and found that the subjects detected the slots more readily when they were parallel to the finger axis.

\section{Acuity and Aging}

A decline in tactile sensory capacity in older subjects has been well documented for different tasks, such as tactile letter recognition (Vega-Bermudez \& Johnson, 2002), grating orientation discrimination (Remblay, Backman, Cuenco, Vant, \& Wassef, 2000), gap detection and point localization (Stevens \& Choo, 1996), and vibratory detec- tion (Gescheider, Bolanowski, Hall, Hoffman, \& Verillo, 1994). The demonstration that skin conformability is the same in older and younger subjects is surprising in view of the changes in skin that occur with aging. Studies that have addressed changes in hairy skin mechanics (we are not aware of comparable studies in glabrous skin) with age suggest that there are changes in skin elasticity, hydration, thickness, and torsion extensibility (Berardesca, Farinelli, Rabbiosi, \& Maibach, 1991; Escoffier et al., 1989). However, our results suggest that these changes do not affect the skin's ability to conform to the spatial features of the stimulus.

The lack of a change in conformability with aging suggests that the loss of tactile spatial acuity is accounted for by some loss in the neural mechanisms leading to perception. Loss of mechanoreceptor density is a possible reason for the loss of mechanoreceptive sensibility (Gescheider et al., 1994; Stevens \& Choo, 1996; Stevens \& Patterson, 1995). Several studies have shown that Pacinian, Meissner, and Merkel receptor density declines with age and that there are morphological changes in the receptors (Bruce,

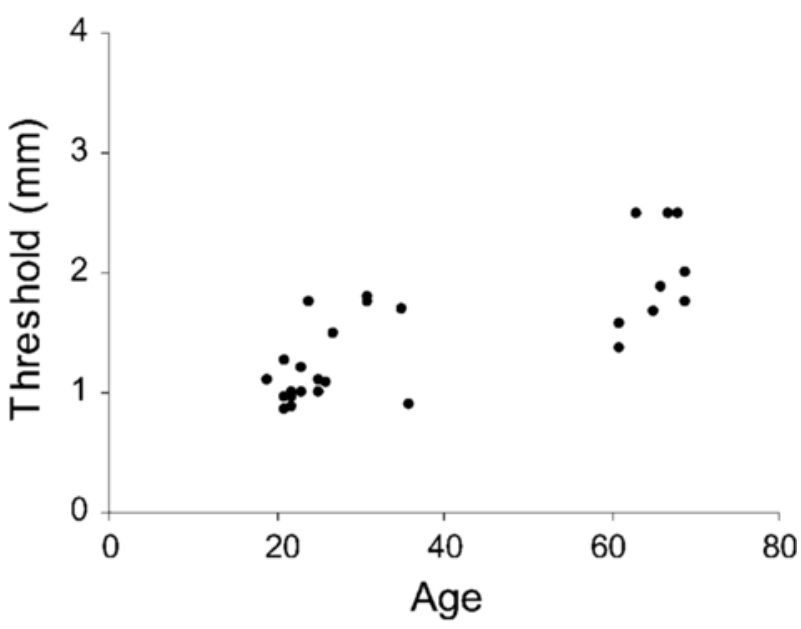

Figure 5. Grating orientation discrimination threshold versus subject age. 


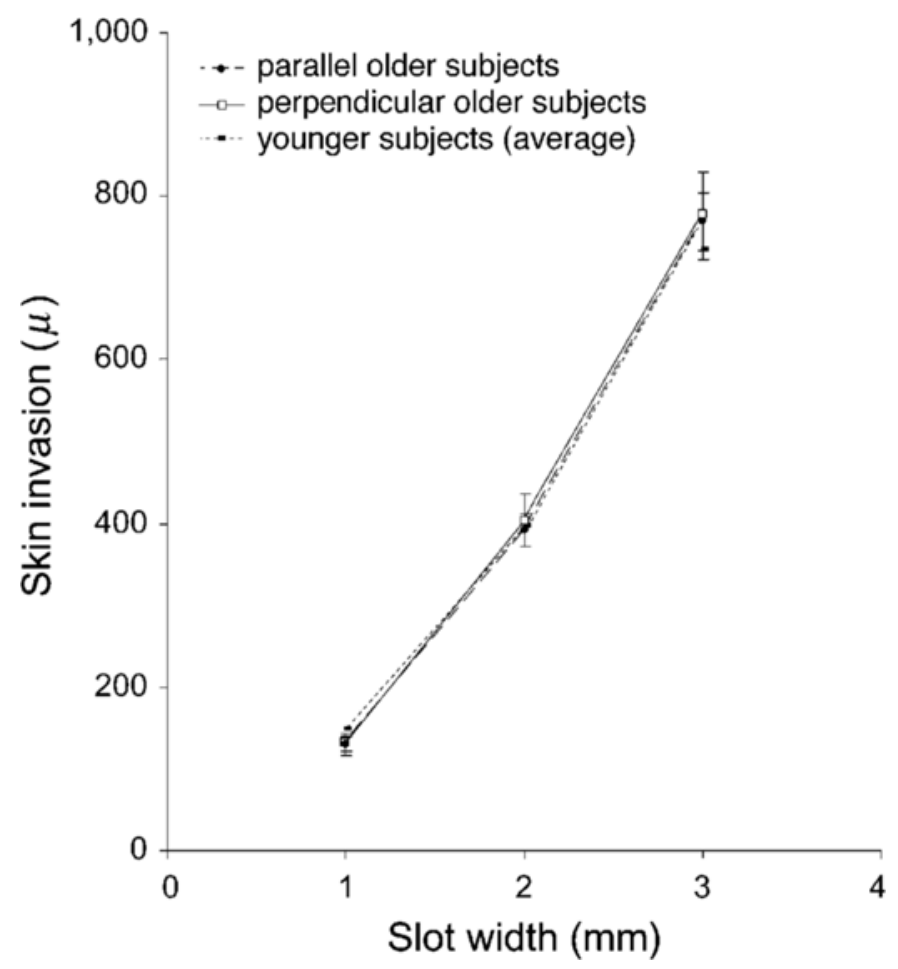

Figure 6. Skin invasion versus slot width for younger and older subjects at $80 \mathrm{~g}$ of force. Dashed lines represent skin invasion when the slot was oriented parallel to the skin ridges; solid lines, when it was perpendicular to the skin ridges. Error bars represent the standard error of the mean for each point.

1980; Cauna, 1965). Since these mechanoreceptors have been linked to the afferent system that transmits tactile information (K. O. Johnson, 2001), one may expect that deterioration in the density of the receptor matrix would lead to deterioration in tactile spatial acuity.

\section{Force and Conformance}

The skin is extremely compliant and conformant. A very light force produces a large indentation (compliance); for example, when a flat surface presses on the skin of a fingerpad, a $30-\mathrm{g}$ force produces $1.5 \mathrm{~mm}$ of skin indentation. Increasing the force more than threefold to $100 \mathrm{~g}$ produces only an extra $0.6 \mathrm{~mm}$ of indentation (VegaBermudez \& Johnson, 1999). The data illustrated in Figure 1 show that the same is true of conformance. The relevant mechanical stimulus for the mechanoreceptors that are responsible for tactile perception is local tissue distortion (Phillips \& Johnson, 1981b), not pressure per se, because they are embedded within an aqueous medium, which is incompressible. The distortion and the accurate representation of spatial form depend on the degree to which the skin conforms to the object being sensed. The finding that the skin is so conformant (that most of the conformation occurs at forces less than $40 \mathrm{~g}$ ) accounts for the fact that neural responses to spatial patterns scanned over the skin are affected minimally by changes in contact force (K. O. Johnson \& Lamb, 1981; Phillips, Johansson, \& John- son, 1992). This, in turn, accounts for the fact that tactile spatial pattern recognition (Loomis, 1985) and roughness judgments (Lederman, 1974) are affected minimally, if at all, by changes in contact force.

\section{Conclusion}

There is a substantial relationship between skin conformance and tactile spatial acuity in the fingertips of younger subjects. However, the general decline in spatial acuity with aging is the result of something other than a change in skin conformance. This suggests that neural factors play the major role in this decline. A secondary finding is that the skin ridges of the fingertip make the skin more conformant in the direction orthogonal to the skin ridges. Therefore, stimuli that vary in the dimension orthogonal to the ridges (e.g., gratings with bars oriented parallel to the ridges) are discriminated more readily than stimuli that vary in the dimension parallel to the ridges (e.g., gratings with bars oriented orthogonal to the ridges).

\section{REFERENCES}

Berardesca, E., Farinelli, N., Rabbiosi, G., \& Maibach, H. I. (1991). Skin bioengineering in the noninvasive assessment of cutaneous aging. Dermatologica, 182, 1-6.

BRUCE, M. F. (1980). The relation of tactile threshold to histology in the fingers of elderly people. Journal of Neurology, Neurosurgery \& Psychiatry, 43, 730-734. 
CaUna, N. (1965). The effects of aging on the receptor organs of the human dermis. In W. Montagna (Ed.), Advances in biology of skin (Vol. 6, pp. 63-96). Elmsford, NY: Pergamon.

CRAIG, J. C. (1999). Grating orientation as a measure of tactile spatial acuity. Somatosensensory \& Motor Research, 16, 197-206.

Craig, J. C., \& Johnson, K. O. (2000). The two-point threshold: Not a measure of tactile spatial resolution. Current Directions in Psychological Science, 9, 29-32.

CrAig, J. C., \& LYLE, K. B. (2001). A comparison of tactile spatial sensitivity on the palm and fingerpad. Perception \& Psychophysics, 63, 337-347.

Escoffier, C., De Rigal, J., Rochefort, A., VAsselet, R., LeVeque, J. L., \& AGACHE, P. G. (1989). Age-related mechanical properties of human skin: An in vivo study. Journal of Investigative Dermatology, 93, 353-357.

Essock, E. A., Krebs, W. K., \& Prather, J. R. (1992). An anisotropy of human tactile sensitivity and its relationship to the visual oblique effect. Experimental Brain Research, 91, 520-524.

Essock, E. A., Krebs, W. K., \& Prather, J. R. (1997). Superior sensitivity for tactile stimuli oriented proximally-distally on the finger: Implications for mixed class 1 and class 2 anisotropies. Journal of Experimental Psychology: Human Perception \& Performance, 23, 515-527.

Gesche IDE R, G. A., Bolanowski, S. J., Hall, K. L., Hoffman, K. E., \& VERILLO, R. T. (1994). The effects of aging on information processing channels in the sense of touch: I. Absolute sensitivity. Somatosensensory \& Motor Research, 11, 345-357.

Johnson, K. L. (1985). Contact mechanics. Cambridge: Cambridge University Press.

JoHnson, K. O. (2001). The roles and functions of cutaneous mechanoreceptors. Current Opinion in Neurobiology, 11, 455-461.

Johnson, K. O., \& LAMB, G. D. (1981). Neural mechanisms of spatial tactile discrimination: Neural patterns evoked by Braille-like dot patterns in the monkey. Journal of Physiology, 310, 117-144.

LEDERMAN, S. J. (1974). Tactile roughness of grooved surfaces: The touching process and the effects of macro- and microsurface structure. Perception \& Psychophysics, 16, 385-395.

Loomis, J. M. (1985). Tactile recognition of raised characters: A parametric study. Bulletin of the Psychonomic Society, 23, 18-20.

Phillips, J. R., Johansson, R. S., \& Johnson, K. O. (1992). Responses of human mechanoreceptive afferents to embossed dot arrays scanned across fingerpad skin. Journal of Neuroscience, 12, 827-839.

PhilliPs, J. R., \& JOHNSON, K. O. (1981a). Tactile spatial resolution: II. Neural representation of bars, edges, and gratings in monkey primary afferents. Journal of Neurophysiology, 46, 1192-1203.

PhILliPs, J. R., \& JoHnson, K. O. (1981b). Tactile spatial resolution: III. A continuum mechanics model of skin predicting mechanorecep- tor responses to bars, edges, and gratings. Journal of Neurophysiology, 46, 1204-1225.

Phillips, J. R., Johnson, K. O., \& BRowne, H. M. (1983). A comparison of visual and two modes of tactual letter resolution. Perception \& Psychophysics, 34, 243-249.

Remblay, F., Backman, A., Cuenco, A., Vant, K., \& Wassef, M. A. (2000). Assessment of spatial acuity at the fingertip with grating (JVP) domes: Validity for use in an elderly population. Somatosensory \& Motor Research, 17, 61-66.

Sathian, K., \& Zangaladze, A. (1996). Tactile spatial acuity at the human fingertip and lip: Bilateral symmetry and inter-digit variability. Neurology, 46, 1464-1466.

Schlereth, T., Magerl, W., \& Rolf-Detlef, T. (2001). Spatial discrimination thresholds for pain and touch in human hairy skin. Pain, 92, 187-194.

SoKal, R. R., \& Rohlf, F. J. (1969). Biometry. San Francisco: Freeman. Stevens, J. C., \& CHOO, K. K. (1996). Spatial acuity of the body surface over the life span. Somatosensensory \& Motor Research, 13, 153-166.

Stevens, J. C., \& PAtterson, M. Q. (1995). Dimension of spatial acuity in the touch sense: Changes of the life span. Somatosensory \& Motor Research, 12, 29-47.

VAN Boven, R. W., \& Johnson, K. O. (1994a). The limit of tactile spatial resolution in humans: Grating orientation discrimination at the lip, tongue, and finger. Neurology, 44, 2361-2366.

VAN BOVEn, R. W., \& Johnson, K. O. (1994b). A psychophysical study of the mechanisms of sensory recovery following nerve injury in humans. Brain, 117, 149-167.

VeGA-Bermude Z, F., \& Johnson, K. O. (1999). SA1 and RA receptive fields, response variability, and population responses mapped with a probe array. Journal of Neurophysiology, 81, 2701-2710.

VeGa-Bermude Z, F., \& Johnson, K. O. (2001). Differences in spatial acuity between digits. Neurology, 56, 1389-1391.

Vega-Bermude Z, F., \& Johnson, K. O. (2002). Spatial acuity after digit amputation. Brain, 125, 1256-1264.

Vega-Bermude Z, F., Johnson, K. O., \& Hsiao, S. S. (1991). Human tactile pattern recognition: Active versus passive touch, velocity effects and patterns of confusion. Journal of Neurophysiology, 65, 531-546.

WhEAT, H. E., \& GoODWIN, A. W. (2000). Tactile discrimination of gaps by slowly adapting afferents: Effects of population parameters and anisotropy in the finger pad. Journal of Neurophysiology, 84, 1430-1444. WoODWARD, K. L. (1993). The relationship between skin compliance, age, gender, and tactile discriminative thresholds in humans. Somatosensory \& Motor Research, 10, 63-67.

(Manuscript received August 1, 2002; revision accepted for publication May 22, 2003.) 